\title{
PREVALENCIA DE HEMATURIA VESICAL ENZOÓTICA BOVINA DETERMINADA MEDIANTE URIANÁLISIS EN OXAPAMPA, PERÚ
}

\author{
Prevalence of Bovine Enzootic Hematuria Detected by Urinalysis \\ in Oxapampa, Peru \\ Karina Herencia B. ${ }^{1,3}$, Néstor Falcón P. ${ }^{1}$, Mario García P. ${ }^{1}$, Alfonso Chavera C. ${ }^{2}$, \\ Christian Gonzáles E. ${ }^{2}$
}

\section{Resumen}

La Hematuria Vesical Enzoótica Bovina (HVEB) es una patología ocasionada por el consumo continuo del helecho común (Pteridium aquilinum). Esta maleza posee un conjunto de sustancias carcinogénicas y mutagénicas, entre las que destaca el ptaquilósido $(\mathrm{Pq})$, responsable del desarrollo de lesiones hemorrágicas y neoplásicas en la vejiga de los bovinos, causando un cuadro progresivo de hematuria. El estudio tuvo como objetivo determinar la prevalencia de HVEB en Chontabamba, provincia de Oxapampa, Perú. Se recolectaron muestras de orina mediante masaje perianal en 210 bovinos hembras, mayores de 2 años de edad, de varios tipos raciales destinados a la producción de carne y leche. Los animales estaban naturalmente expuestos al consumo de helecho común. Las muestras fueron analizadas mediante la tira reactiva urinaria (TRU) y la evaluación microscópica de sedimento urinario (EMSU). Se encontró una prevalencia de 7.6 $\pm 3.6 \%$ mediante TRU y $15.2 \pm 4.9 \%$ mediante EMSU. Asimismo, se encontró una mayor prevalencia en animales de 1.5 a 4 años de edad. No se encontró asociación estadística entre presencia de HVEB con grupo racial o fin productivo. Se concluye que la zona de estudio es endémica a HVEB.

Palabras clave: hematuria, pteridium aquilinum, bovino, urianálisis, prevalencia

\section{Abstract}

Bovine Enzootic Vesical Hematuria (BEVH) is a disease caused by continuous consumption of the common fern (Pteridium aquilinum). This plant has a set of carcinogenic and mutagenic substances, including the ptaquiloside (pq) which is responsible for neoplastic and hemorrhagic lesions in the bladder mucosa of cattle, eventually leading to hematuria. This study determined the prevalence of BEVH in cattle at Chontabamba, province of Oxapampa, Peru. Urine samples were collected by perianal massage from 210 females older than 2 years old of various genotypes and productive

\footnotetext{
${ }^{1}$ Facultad de Veterinaria y Zootecnia, Universidad Peruana Cayetano Heredia, Lima

${ }^{2}$ Facultad de Medicina Veterinaria, Universidad Nacional Mayor de San Marcos, Lima

${ }^{3}$ E-mail: elianaherencia@gmail.com
} 
purpose (milk and beef). The animals were naturally exposed to consumption of the common fern. Urine samples were subject to urianalysis test involving the application of urine test strips (UTS) and microscopic examination of urinary sediment (MEUS). The prevalence of BEVH was $7.6 \pm 3.6 \%$ by UTS and $15.2 \pm 4.9 \%$ by MEUS. The prevalence was higher in 1.5 to 4 year old animals. There was no statistical association between presence of $\mathrm{BEVH}$ with breed or productive purpose. It is concluded that this geographic area is endemic to BEVH.

Key words: hematuria, Pteridium aquilinum, bovine, urianalysis, prevalence

\section{INTRODUCCIÓN}

La Hematuria Vesical Enzoótica Bovina (HVEB) es una enfermedad no infecciosa, de mortalidad elevada, que se caracteriza principalmente por la presencia de procesos tumorales de formas y tamaños variables en la pared de la vejiga (Radostits et al., 2002). Su desarrollo está asociado a la ingestión continua y prolongada de variedades de Pteridium aquilinum $(\mathrm{Pa})$, denominadas Arachnoideum y Caudatum (AlonsoAmelot, 1999), donde el principal signo clínico es la hematuria (Marrero et al., 2001). Además, tiene relación con agentes infecciosos como los Papillomavirus y Citomegalovirus (Sánchez-Villalobos, 2006).

Afecta a vacunos mayores de un año de edad (Radostits et al., 2002) en crianza al pastoreo en zonas húmedas y con suelos ácidos localizados entre 1000 a $2500 \mathrm{msnm}$ (Alonso-Amelot, 1999; Sánchez-Villalobos et al., 1999; Sánchez-Villalobos, 2006) y de baja fertilidad (Radostitis et al., 2002), por lo que la presencia de HVEB en la finca resulta ser un indicador de mal manejo de potreros. La raza del animal no es un factor de riesgo (Pamukcu, 1974; Villafañe y Lichtenberger, 1979). El Pa produce una amplia gama de sustancias mutagénicas y carcinogénicas, entre los cuales destaca el ptaquilósido, por lo que la enfermedad también es denominada «Intoxicación por ptaquilósido» (Radostits et al., 2002).
La HVEB se inicia con el consumo prolongado de frondes de $\mathrm{Pa}$. Parte del ptaquilósido es destruida en el abomaso por la acción de ácidos y enzimas digestivas, pero otra parte llega al intestino delgado, donde a través de las vellosidades intestinales, ingresa al torrente sanguíneo y se disemina al hígado, riñón, músculo y vejiga urinaria, entre otros órganos (Alonso-Amelot, 1999). El ptaquilósido en medios alcalinos ( $\mathrm{pH}$ de vejiga), se transforma en una dienona inestable, tóxico verdadero (Radostits et al., 2002), que posee gran afinidad con proteínas con terminales aminos expuestos (entre ellos el ADN), provocando alteraciones permanentes en los genes, inhibiendo a la proteína P53, encargada de regular la apoptosis. Ello lleva a la aparición de lesiones neoplásicas de tipo epitelial, no epitelial (endotelial) o mixtas (AlonsoAmelot y Avendaño, 2002), principalmente en vejiga y en el aparato digestivo (AlonsoAmelot, 1999).

Las neoplasias de tipo endotelial ocasionan úlceras hemorrágicas en la mucosa vesical (Moulton, 1978), que provocan microhematuria (signo subclínico), que luego se transforma en macrohematuria (signo clínico) (Jubb et al., 1991). La hemorragia vesical causa una depresión de la médula ósea, pancitopenia, proteinuria y piuria variable (Peña et al., 2001). El cuadro continúa con hipoxia cerebral, shock hipovolémico, paro cardiorrespiratorio y muerte del animal (Radostits et al., 2002). 
La prevalencia de HVEB no es alta, dada su naturaleza no infecciosa, pero la merma en producción causa pérdidas económicas importantes (Radostits et al., 2002). Asimismo, el ptaquilósido es excretado por la glándula mamaria (Alonso-Amelot y Avendaño 2002), representando un riesgo para el hombre, pues su consumo se ha relacionado con una mayor probabilidad de ocurrencia de cáncer gástrico y de colon (Villalobos, 1985).

Se han reportado prevalencias menores a $1 \%$ en EEUU y Kenya (Pamukcu, 1974), menores de $20 \%$ en Bolivia, China, Colombia, India, Nepal, Portugal, Venezuela (De Jongh, 1978, Villafañe y Litchtenberger, 1979; Hopkins 1987; Xu, 1992; Marrero et al., 2001; Dawra et al., 2002; Pinto et al., 2004), y más del $40 \%$ en Brasil, España, Turquía y Yugoslavia (Butozan, 1935; Pamukcu et al., 1976; Peña et al., 2000; Franca et al., 2002). En un estudio más reciente, SánchezVillalobos et al. (2006) en Mérida, Venezuela, hallaron $16 \%$ de prevalencia mediante urianálisis y necropsia.

En el Perú, el primer reporte oficial de HVEB se realizó a través de un estudio anátomo-histopatológico de órganos de animales con antecedentes clínicos de hematuria en la provincia de Oxapampa (Gonzáles, 2003). Asimismo, Verde (2009), en un estudio de diagnóstico clínico mediante urianálisis realizado en el distrito de Oxapampa, reportó una prevalencia de $26.1 \%$.

No existen pruebas de laboratorio para la identificación del ptaquilósido en leche o sangre. Por ello, el urianálisis compuesto de la Tira Reactiva Urinaria (TRU) como prueba tamiz, y la Evaluación Microscópica del Sedimento Urinario (EMSU) como prueba confirmatoria (Sánchez-Villalobos, 2006) resultan una alternativa confiable, siempre que estén aunadas a información clínica del animal (Verde, 2009). La EMSU tiene una sensibilidad de $88.3 \%$ y una especificidad de
99.2\% y ha demostrado su practicidad clínica para identificar bovinos en fase subclínica (microhematuria) (Sánchez-Villalobos, 2006; Verde, 2009). Las pruebas del urianálisis, permiten determinar hematuria y diferenciarla de otras condiciones clínicas o como hemoglobinuria en la piroplasmosis (Radostits et al., 2002). La presencia de un número mayor o igual a cinco glóbulos rojos por campo en el sedimento urinario se considera hematuria, donde Verde (2009) determina tres grados de hematuria $(I=5-100$ eritrocitos por campo; $\mathrm{II}=100-1000$; III > 1000).

La hematuria es un signo inespecífico para una evaluación diagnóstica, de modo que un caso positivo de hematuria es considerado HVEB cuando el diagnóstico clínico se complementa con una evaluación poblacional de los animales y la inspección general del establecimiento, con énfasis en los potreros (presencia de Pa) (Verde, 2009). No obstante, el diagnóstico definitivo de la HVEB se obtiene en la necropsia y con histopatología (Radostits et al., 2002).

El presente estudio tuvo por objetivo determinar la prevalencia de HVEB en fincas ganaderas de la provincia de Oxapampa, Pasco, Perú.

\section{Materiales y Métodos}

Se trabajó durante el mes de octubre de 2008 con ganado bovino de crianza extensiva y semiextensiva en una zona endémica a $\mathrm{Pa}$ en el distrito de Chontabamba, provincia de Oxapampa (Pasco). Los animales fueron Bos taurus, Bos indicus y sus cruces, hembras, mayores de 18 meses de edad, que fueron distribuidos en tres grupos etarios $(<2,2$ a $4, y>4$ años de edad). Los animales fueron seleccionados en forma aleatoria entre los ganaderos que aceptaron participar en el estudio, hasta recolectar el total de muestras requeridas por el estudio. El estudio fue observacional, descriptivo y transversal. 
El cálculo del tamaño de muestra se realizó utilizando la fórmula de comparación de una proporción, tomando una prevalencia referencial de 16\% (Sánchez-Villalobos et al., 2006), con un nivel de confianza del $95 \%$ $(Z=1.96)$ y un error máximo admisible de $5 \%$. El tamaño de muestra resultante fue de 210 bovinos.

Para la toma de la muestra de orina, los animales se inmovilizaron en un brete o manga, y se empleó el masaje perineal para estimular la micción (Sánchez-Villalobos, 2006). La orina se recolectó en frascos de plástico limpios, rotulados, de boca ancha y tapa rosca $(200 \mathrm{ml})$. Asimismo, se obtuvo información clínica de los animales y de las condiciones de la finca a través de una ficha epidemiológica.

El procesamiento de las muestras de orina se llevó a cabo en un laboratorio clínico implementado en la ciudad de Oxapampa, donde se emplearon las siguientes pruebas:

TRU: Utilizando la tira reactiva urinaria (Combur Test ${ }^{\circledR}$, Roche, EEUU). El criterio de diagnóstico para definir a un animal positivo a hematuria fue la observación de cambio de coloración.

EMSU: La evaluación microscópica del sedimento urinario fue considerada como la prueba de oro confirmatoria. El sedimento se obtuvo por centrifugación de la orina, y mediante observación directa al microscopio se identificó y cuantificó la presencia de glóbulos rojos.

Se determinó la prevalencia de la HVEB e intervalo de confianza del $95 \%$ de la HVEB para cada prueba diagnóstica. Asimismo, se analizó frecuencia de presentación de la HVEB en los estratos de las variables tipo racial, estrato etario y propósito de producción (leche y carne), mediante la prueba de Chi cuadrado. Asimismo, se analizó la concordancia de pruebas diagnósticas mediante el Índice de Kappa y la prueba de McNemar.

\section{Resultados}

Se encontró una prevalencia de $7.6 \pm$ $3.6 \%$ (16/210) utilizando la técnica de la TRU y de $15.2 \pm 4.9 \%(32 / 210)$ con la EMSU, habiendo diferencia estadística $(\mathrm{p}<0.05)$ entre los resultados de ambas pruebas diagnósticas (Cuadro 1).

El análisis de los resultados de la HVEB según estrato etario encontró una mayor frecuencia de positivos en animales menores de cuatro años, siendo los resultados consistentes para ambas pruebas diagnósticas; sin embargo, sólo se observó diferencia estadística para la EMSU (p<0.05) (Cuadro 1). Por otro lado, no se encontró diferencias en prevalencia de HVEB según el tipo racial (Bos taurus, $\mathrm{n}=179$; Bos indicus y cruces, $\mathrm{n}=31$ ), ni entre el fin productivo (leche, $n=188$; carne, $n=22$ ). Asimismo, la prevalencia de HVEB fue muy variable dentro de cada finca (Cuadro 2).

La prueba de EMSU mostró un mayor número de animales positivos a hematuria en grado I en relación a la TRU (Cuadro 3).

Los resultados de ambas pruebas diagnósticas muestran una concordancia de 63\% mediante el Índice de Kappa, e indican a su vez que dichas pruebas no pueden ser reemplazables mutuamente $(\mathrm{p}<0.05)$ mediante la prueba de Chi cuadrado de McNemar. La discrepancia surgió cuando en el diagnóstico de TRU se reporta 16 animales negativos, los cuales fueron considerados como positivos a la EMSU (Cuadro 4).

\section{Discusión}

Se encontró una diferencia significativa en la prevalencia de HVEB cuando se empleó la prueba TRU (7.6\%) y la EMSU (15.2\%) al analizar las 210 muestras de orina. Este resultado es comparable con los hallazgos de Verde (2009), quien halló prevalencias de 13.3 y $26.1 \%$ en 211 anima- 
Cuadro 1. Frecuencias de Hematuria Vesical Enzoótica Bovina a través de la Tira Reactiva Urinaria (TRU) y la Evaluación Microscópica del Sedimento Urinario (EMSU), según la edad de las vacas (Pasco, Perú, 2008)

\begin{tabular}{cccccc}
\hline \multirow{2}{*}{$\begin{array}{c}\text { Edad } \\
\text { (años) }\end{array}$} & $\begin{array}{c}\text { Muestras } \\
(\mathrm{n})\end{array}$ & \multicolumn{2}{c}{ Positivos a TRU } & \multicolumn{2}{c}{ Positivos a EMSU } \\
\cline { 3 - 6 } & 20 & 3 & $15.0^{\mathrm{a}}$ & 5 & $25.0^{\mathrm{a}}$ \\
\hline $1.5 \mathrm{a} 2$ & 66 & 8 & $12.1^{\mathrm{a}}$ & 17 & $25.8^{\mathrm{a}}$ \\
$>2 \mathrm{a} 4$ & 124 & 5 & $4.0^{\mathrm{a}}$ & 10 & $8.1^{\mathrm{b}}$ \\
$>4$ & 210 & 16 & $7.6^{1}$ & 32 & $15.2^{2}$ \\
\hline Total & &
\end{tabular}

$a, b$ Letras diferentes dentro de columnas indican diferencias estadísticas $(p<0.05)$

${ }^{1,2}$ Números diferentes en los totales entre pruebas diagnósticas indican diferencias estadísticas $(p<0.01)$

Cuadro 2. Frecuencia de presentación de Hematuria Vesical Enzoótica Bovina en fincas ganaderas de Chontabamba, provincia de Oxapampa (Pasco), determinado mediante la Evaluación Microscópica del Sedimento Urinario (EMSU)

\begin{tabular}{clc}
\hline Finca & Tipo de crianza & Frecuencia (\%) \\
\hline 1 & Semi-extensivo & 7.0 \\
2 & Extensivo & 48.0 \\
3 & Extensivo & 8.0 \\
4 & Extensivo & 0.0 \\
5 & Extensivo & 0.0 \\
6 & Extensivo & 3.9 \\
7 & Extensivo & 5.3 \\
8 & Semi-extensivo & 20.0 \\
\hline
\end{tabular}

Cuadro 3. Distribución de los grados de Hematuria Vesical Enzoótica Bovina en base a los resultados de la Tira Reactiva Urinaria (TRU) y la Evaluación Microscópica del Sedimento Urinario (EMSU)

\begin{tabular}{cccccc}
\hline \multirow{2}{*}{$\begin{array}{c}\text { Grados de } \\
\text { hematuria }\end{array}$} & $\begin{array}{c}\text { Muestras } \\
(\mathrm{n})\end{array}$ & \multicolumn{2}{c}{ Positivos a TRU } & \multicolumn{2}{c}{ Positivos a EMSU } \\
\cline { 3 - 6 } & 178 & 0 & 0.0 & 0 & 0 \\
\hline Normal & 16 & 3 & 18.6 & 16 & 100.0 \\
I & 9 & 6 & 66.7 & 9 & 100.0 \\
II & 7 & 7 & 100.0 & 7 & 100.0 \\
III & & & & $\%$ & $\mathrm{n}$ \\
\hline
\end{tabular}

I =5-100 eritrocitos por campo; II =100-1000 eritrocitos por campo; III >1000 eritrocitos por campo 
Cuadro 4. Diagnóstico comparativo de la Hematuria Vesical Enzoótica Bovina en base a los resultados de la Tira Reactiva Urinaria (TRU) y la Evaluación Microscópica del Sedimento Urinario (EMSU)

\begin{tabular}{lcccc}
\hline & & \multicolumn{2}{c}{ TRU } & \multirow{2}{*}{ Total } \\
\cline { 3 - 4 } & & Positivos & Negativos & \\
\hline \multirow{2}{*}{ EMSU } & Positivos & 16 & 16 & 32 \\
& Negativos & 0 & 178 & 178 \\
\hline \multirow{2}{*}{ Total } & & 16 & 194 & 210 \\
\hline
\end{tabular}

les, utilizando las pruebas de TRU y EMSU, respectivamente. Ambos estudios difieren con los resultados de Sánchez-Villalobos et al. (2006), donde la prevalencia de $88 \%$ con TRU y de $97.9 \%$ con EMSU fueron bastante similares, considerando que ambas pruebas presentan altos grados de especificidad y sensibilidad durante la fase subclínica de la enfermedad.

La prueba EMSU presenta una mayor sensibilidad al ser una prueba de observación directa, frente a TRU que es una prueba química o indirecta, que está sujeta a factores externos como residuos de desinfectantes, y el elevado $\mathrm{pH}$, alto peso específico y valores de moderada a alta concentración de ácido ascórbico en la orina, entre otros, los cuales pueden inhibir la formación del color o disminuir la sensibilidad para detectar los glóbulos rojos (Verde, 2009).

No obstante las diferencias encontradas entre ambos métodos, el empleo simultáneo de TRU y EMSU mostró ser de gran utilidad para detectar casos subclínicos (microhematuria). TRU otorga cierta aproximación a un caso positivo, con la ventaja de que puede emplearse directamente en campo y su manejo es sencillo. Las muestras positivas a TRU podrían, entonces, ser confirmadas con EMSU de existir un laboratorio clínico en la zona. Adicionalmente, se debe considerar que el producto comercial TRU utilizado fue de uso humano (Combur Test ${ }^{\circledR}$,
Roche, USA), aunque de un laboratorio distinto a los empleados por Sánchez-Villalobos et al. (2006a) (Combi-Screen ${ }^{\circledR}$, 10SL) y Verde (2009) (SD UroColor ${ }^{\mathrm{TM}}$ 11S, Standardia, Korea).

La EMSU viene a ser la prueba de oro para el diagnóstico clínico de la HVEB y, por lo tanto, la prueba de elección para la determinación de la prevalencia de la enfermedad, lo más cercana a la real, que solo se lograría con necropsia. Pese a la baja sensibilidad observada en la TRU, el resultado no deja de ser interesante para una primo-evaluación de muestras de orina en el diagnóstico de la HVEB. Posiblemente, en estudios futuros, se deberían emplear marcas reportadas con mejores resultados o utilizar una tira específica para bovinos (Combi-Screen Vet 11, Analyticon Biotechnologies, Germany).

La prevalencia encontrada en el presente estudio fue diferente a la reportada por Verde (2009), quien encuentra $26.1 \%$ de animales afectados en el distrito de Oxapampa, contiguo a Chontabamba, lugar del presente estudio, y utilizando el mismo método diagnóstico. Las diferencias podrían ser explicadas por tratarse de años y épocas distintos, dado que el primer estudio se hizo al inicio de la época de seca (mayo de 2007), mientras que el presente trabajo fue realizado al final de la época de seca (octubre de 2008), donde hay grandes diferencias en la 
disponibilidad de pastos frente al helecho, así como una posible variación en el nivel de toxicidad de esta maleza.

La HVEB es una enfermedad crónica, de lento desarrollo, pues depende del nivel de consumo del helecho tóxico. Estudios previos demuestran que animales mayores de 2-3 años tienen mayor riesgo de presentar la HVEB (Pamukcu, 1974; De Jongh, 1978; Sánchez Villalobos et al., 1999). No obstante, en el presente estudio, animales entre 1.5 a 2 años de edad presentaron un porcentaje similar de prevalencia que animales de 2- 4 años. Este hallazgo podría indicar una excesiva disponibilidad de brotes de helechos tóxicos al alcance de los animales más jóvenes del hato, así como un alto contenido de iludanos en el Pa de la zona, que estarían impactando en la salud de los animales. Por otro lado, los grupos etarios no fueron homogéneos en cantidad, por lo que no se pueden realizar mayores conclusiones.

La ausencia de asociación estadística entre casos de HVEB, con el grupo racial coincide con resultados publicados por diversos autores (Pamukcu, 1974; Villafañe y Litchtenberger, 1979; Sánchez-Villalobos et al., 1999). Asimismo, las prevalencias similares entre el ganado lechero y el ganado de carne encontrado en el presente estudio no permiten obtener datos conclusivos, dado que la mayor parte de la población muestreada fue de tipo lechero; no obstante, Villafañe y Lichtenberger (1979) mencionan que la HVEB afecta mayormente en ganado lechero.

Los ganaderos de la zona tienen conocimiento del problema que representa la HVEB en sus rebaños, de modo que algunos toman las provisiones necesarias para limitar el crecimiento del helecho en los potreros y para descartar tempranamente a los animales que muestren signos de hematuria. Estas diferencias entre fincas en el manejo animal podría explicar las notables diferencias en términos de prevalencia de la HVEB, diferencias que incluso de observan dentro del mismo tipo de crianza (semiextensivo y extensivo).

\section{ConClusiones}

- La prevalencia de HVEB en las fincas ganaderas de la zona de Chontabamba, Pasco, fue $7.6 \pm 3.6 \%$ (16/210) mediante la técnica de TRU y de $15.2 \pm 4.9 \%$ (32/210) con EMSU, siendo el valor obtenido con EMSU el más aproximado a la realidad, debido a la mayor sensibilidad de la prueba.

- Se encontró diferencias en la prevalencia de HVEB por efecto del grupo etario, mas no por el tipo racial o el fin productivo (carne, leche) del ganado.

- Se debe considerar al distrito de Chontabamba (provincia de Oxapampa) como zona endémica a la HVEB.

\section{Literatura Citada}

1. Alonso-Amelot ME, Avendaño M. 2002. Human carcinogenesis and bracken fern: a review of the evidence. Curr Med Chem 9: 675-686.

2. Alonso-Amelot ME. 1999. Helecho macho, salud animal y salud humana. Rev Fac Agron LUZ 16: 528-541.

3. Butozan V. 1935. Chronishe haematurie bei den ridern in der Vrabaska banovina. Jugoslav Vet Glasnik 15: 225-233.

4. Dawra R, Kurade N, Sharma O. 2002. Carcinogenicity of the fern Pteridium aquilinum collected from enzootic bovine haematuria free-hilly in India. Curr Sci 83: 1005-1009.

5. De Jongh F. 1978. Ocurrencia y diagnóstico histopatológico de la hematuria vesical clínica en bovinos de la zona alta del estado Mérida. Corpoandes-UCV 10: 1-22.

6. Franca T, Tokarnia C, Peixoto P. 2002. Enfermidades determinadas pelo princípio radiomimético de Pteridium aquilinum (Polypodiaceae). Pesq Vet Bras 22(3): 85-95.

7. Gonzáles C. 2003. Frecuencia y caracterización anátomo-histopatológica de las lesiones encontradas en bovinos con 
hematuria en Oxapampa, Pasco. Tesis de Médico Veterinario. Lima: Universidad Nacional Mayor de San Marcos. 84 p.

8. Hopkins N. 1987. Enzootic haematuria in Nepal. Trop Anim Hlth Prod 19: 159164.

9. Jubb K, Kennedy P, Palmer N. 1991. Pathology of domestic animals. Vol II. $4^{\text {th }}$ ed. California: Academic Press. 699 p.

10. Marrero E, Bulnes $C$, Sánchez L, Palenzuela I, Stuart R, Jacobs, Romero J. 2001. Caracterización general del cuadro de toxicosis por Pteridium aquilinum en bovinos en el departamento de Tarija, Bolivia. Rev Salud Anim 23(1): 16-22.

11. Moulton J. 1978. Tumors in domestic animals. $2^{\text {nd }}$ ed. California, USA: University of California. $453 \mathrm{p}$.

12. Pamukcu A. 1974. Tumors of the urinary bladder. In: International histological classification of tumors of domestic animals. Bull Wld Hlth Org 50(4): 43-52.

13. Pamukcu A, Price J, Bryan G. 1976. Naturally occurring and bracken ferninduced bovine urinary bladder tumors. Vet Pathol 13: 110-122.

14. Peña L, Rodríguez A, Blanco J, Pérez M, González M, Sánchez M, Pizarro M, et al. 2001. Hallazgos clínicos y anatomopatológicos de la hematuria enzoótica bovina en una explotación Avileña-Negra Ibérica. En: VII Congreso Internacional de Medicina Bovina. Madrid, España. p 34-41.

15. Pinto C, Januário T, Geraldes M, Machado J, Lauren DR, Smith BL, Robinson RC. 2004. Bovine enzootic haematuria on São Miguel Island - Azores. Acamovic CS 85: 564-574.

16. Radostits O, Gay C, Blood D, Hinchcliff K. 2002. Enfermedades causadas por fitotoxinas mayores. En: Tratado de las enfermedades del ganado bovino, ovino, porcino, caprino y equi-

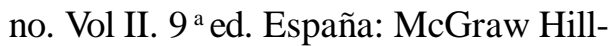
Interamericana. p 1971-1973.

17. Sánchez-Villalobos A, García D, De Alvarado Cruz, Villegas M, Vilarroel $R$, Jiménez U. 1999. Factores de riesgo asociados con hematuria enzoótica bovina en el municipio Campo Elías, estado Mérida, Venezuela. Rev Cienc FCV-LUZ 9: 378-387.

18. Sánchez-Villalobos A, Árraga de Alvarado C, Villaruel R, Pino D, García D, Sánchez G. 2006. Validez, seguridad y cociente de verosimilitud de los métodos de tiras reactivas para orina y examen microscópico del sedimento urinario en el diagnóstico de la hematuria vesical enzoótica bovina. Rev Cien FCVLUZ 16: 604-612.

19. Sánchez-Villalobos A. 2006. Importancia de la toxicosis para la salud humana. En: Hematuria enzoótica bovina. Maracaibo: Grafifor. p 79-86.

20. Verde G 2009. Diagnóstico clínico de la hematuria vesical enzoótica bovina por urianálisis en vacunos de la provincia de Oxapampa, Pasco. Tesis de Médico Veterinario. Lima: Universidad Peruana Cayetano Heredia. 25 p.

21. Villalobos J. 1985. Carcinogenicidad del Pteridium aquilinum y alta incidencia del cáncer gástrico de Costa Rica. Rev Cost Cien Med 6(3): 131-139.

22. Villafañe F, Lichtenberger E. 1979. Hematuria vesical bovina (HVB). ACOVEZ 3(11): 9-13.

23. Xu L. 1992. Bracken poisoning and enzootic haematuria in cattle in China. Res Vet Sci 53: 116-121. 